\title{
DNA damage induces reactive oxygen species generation through the H2AX-Nox1/Rac1 pathway
}

\author{
MA Kang ${ }^{1,2}$, E-Y So ${ }^{2}$, AL Simons $^{3}$, DR Spitz ${ }^{3}$ and T Ouchi ${ }^{\star * 1,2,4}$
}

The DNA damage response (DDR) cascade and ROS (reactive oxygen species) signaling are both involved in the induction of cell death after DNA damage, but a mechanistic link between these two pathways has not been clearly elucidated. This study demonstrates that ROS induction after treatment of cells with neocarzinostatin (NCS), an ionizing radiation mimetic, is at least partly mediated by increasing histone H2AX. Increased levels of ROS and cell death induced by H2AX overexpression alone or DNA damage leading to $\mathrm{H} 2 \mathrm{AX}$ accumulation are reduced by treating cells with the antioxidant N-Acetyl-L-Cysteine (NAC), the NADP(H) oxidase (Nox) inhibitor DPI, expression of Rac1N17, and knockdown of Nox1, but not Nox4, indicating that induction of ROS by H2AX is mediated through Nox1 and Rac1 GTPase. H2AX increases Nox1 activity partly by reducing the interaction between a Nox1 activator NOXA1 and its inhibitor 14-3-3zeta. These results point to a novel role of histone H2AX that regulates Nox1-mediated ROS generation after DNA damage.

Cell Death and Disease (2012) 3, e249; doi:10.1038/cddis.2011.134; published online 12 January 2012

Subject Category: Experimental Medicine

DNA double-strand breaks (DSBs) can cause genetic mutations that activate oncogenes, inactivate tumor suppressors, and change the levels or functions of 'modifier' proteins, which determine chemosensitivity or tumor progression, thus potentially increasing an organism's susceptibility to cancer and a myriad of other diseases. ${ }^{1,2}$ To defend themselves against the deleterious effects of DSBs, eukaryotic organisms employ an intricate DNA damage response (DDR) pathway to detect DNA lesions, arrest the cell cycle until damaged DNA is repaired, and to induce cell death if the lesions are overwhelmed. $^{1-3}$ Thus, efficient induction of the DDR and cell death pathways after DNA damage is crucial in cellular defenses against malignant transformation.

One of the most important proteins in the DDR pathway is histone $\mathrm{H} 2 \mathrm{AX} .{ }^{4,5}$ Results from H2AX knockout studies in mice indicate that loss of one or two copies of the H2AX gene compromises genomic integrity and DDR efficiency and increases tumor formation in a p53-null background. ${ }^{6}$ Furthermore, H2AX phosphorylation status has been shown to determine whether cells repair the damaged DNA to survive or undergo apoptosis. ${ }^{7}$ In response to DNA DSBs, ATM and/ or DNA-PK phosphorylate histone $\mathrm{H} 2 \mathrm{AX}$ at Ser139 to form $\gamma \mathrm{H} 2 \mathrm{AX}{ }^{8}$ Formation of $\gamma \mathrm{H} 2 \mathrm{AX}$ foci on DSB sites is the earliest and the critical event in the DDR pathway. ${ }^{4,5,9-11}$ H2AX not only serves to indicate the localization of DNA lesions, ${ }^{10}$ but its phosphorylation and subsequent ubiquitylation by the RNF8 ubiquitin ligase are required for DNA damage signal amplification and the accumulation of numerous DDR proteins at the sites of DSBs to form the so-called ionizing radiation-induced foci. ${ }^{4,5,9,12}$

Another important determinant of genomic integrity and cellular response to DNA damage is the level of intracellular reactive oxygen species (ROS), which is tightly regulated through the coordinated activities of cellular pro-oxidants and antioxidants. Intracellular ROS can act as a cellular toxicant or a signaling molecule, depending on its concentration and localization. Intracellular ROS is primarily generated through aerobic metabolism or through a specialized group of enzymes, collectively known as the $\mathrm{NAD}(\mathrm{P}) \mathrm{H}$ oxidases (Noxes). Human cells possess seven distinct Nox enzymes including: Nox1-5 and Duox1-2 (reviewed in ${ }^{13-15}$ ). Among them is Nox1 that is expressed in epithelial cells and nonphagocytes. At the molecular level, Nox1 associates with the membrane subunit $\mathrm{p} 22^{\text {phox }}$, which is necessary for enzymatic activity. ${ }^{16}$ Nox 1 is activated by forming a complex with the cytoplasmic activators $\mathrm{p} 47^{\text {phox }}$ and $\mathrm{p} 67^{\text {phox }}$ (and their nonphagocytic homologs NoxO1 and NoxA1) ${ }^{17}$ and the small GTPase Rac1. ${ }^{13,18}$ The primary ROS produced by Nox1 is $\mathrm{O}_{2} \cdot{ }^{-}$, although $\mathrm{H}_{2} \mathrm{O}_{2}$ is thought to be the most important signaling molecule in Nox1 signal transduction. Importantly, Nox1-induced ROS has been implicated in oncogenic signaling in Ras-transformed NIH3T3 cells, where depletion of $\mathrm{H}_{2} \mathrm{O}_{2}$ suppressed uncontrolled cell growth. ${ }^{19}$

DNA damage from various sources has been shown to increase ROS levels. ${ }^{20}$ DNA damage-induced ROS is important in the regulation of cell death and survival, ${ }^{21,22}$

\footnotetext{
${ }^{1}$ Department of Molecular Biosciences, IBIS Program, Northwestern University, Evanston, IL 60201, USA; ${ }^{2}$ Department of Medicine, Systems Biology Program, NUHS, University of Chicago, Evanston, IL 60201, USA; ${ }^{3}$ Free Radical and Radiation Biology Program, Department of Radiation Oncology, Holden Comprehensive Cancer Center, The University of lowa, lowa City, IA 52242, USA and ${ }^{4}$ Department of Cancer Genetics, Roswell Park Cancer Institute, Buffalo, NY 14263, USA

${ }^{*}$ Corresponding author: T Ouchi, NUHS, University of Chicago, 1001 University Place, Evanston, IL 60201, USA. Tel: + 224 364 7687; E-mail: touchi@ bsd.uchicago.edu or Department of Cancer Genetics, Roswell Park Cancer Institute, Elm and Carlton Streets, Buffalo, NY 14263, USA. Tel: + 7168457173 ; Fax: 716845 1698; E-mail: Toru.Ouchi@RoswellPark.org

Keywords: DNA damage; H2AX; Nox1; Rac1; ROS

Abbreviations: DDR, DNA damage response; ROS, reactive oxygen species; NCS, neocarzinostatin; NAC, N-Acetyl-L-Cysteine; Nox, NADP(H) oxidase; DSBs, DNA double-strand breaks; ATM, ataxia telangiectasia mutated

Received 27.10.11; revised 29.11.11; accepted 01.12.11; Edited by RA Knight
} 
partly due to its ability to regulate p53 activity. ${ }^{23,24}$ In turn, the tumor suppressor p53 has been demonstrated to regulate basal and DNA-damage-induced ROS levels. ${ }^{24-26}$ However, the exact mechanism by which DNA damage induces ROS and the involvement of other DDR proteins in ROS generation remain to be elucidated.

In this study, we demonstrate that DNA damage induces ROS through histone H2AX, Nox1, and Rac1. DNA damageinduced ROS is significantly increased by $\mathrm{H} 2 \mathrm{AX}$ overexpression and reduced by its knockdown. ROS induction by histone H2AX expression can be abrogated by treatment of cells with the Nox inhibitor diphenyliodonium chloride (DPI), expression of a dominant negative Rac1 mutant (Rac1N17), and knockdown of Nox1. Recent studies have demonstrated that 14-33zeta binds to NoxA1, resulting in the inactivation of Nox1 by sequestration of NOXA1 from Nox1 in the plasma membrane. ${ }^{27}$ More recent proteomic analysis indicated that H2AX constitutively binds to 14-3-3zeta in a normally growing human hepatocellular carcinoma cell line. ${ }^{28}$ We found that 14-3-3zeta forms a complex with NOXA1 in control U2OS cells, and that $\mathrm{H} 2 \mathrm{AX}$ overexpression reduces this interaction. We also found that DNA damage generates an oscillation of increased ROS, and that suppression of DNA damageinduced ROS by the antioxidant N-Acetyl-L-Cysteine (NAC) reduces apoptosis. Our results point to a novel interaction between H2AX and Nox1, which regulates ROS levels and apoptosis after DNA damage.

\section{Results}

ROS induction after DNA damage in mammalian cells. DNA damage has been shown to stimulate the production of ROS in cell cultures. ${ }^{20,29,30}$ We first examined how different DNA-damaging agents induce ROS in different mammalian cell lines. Sub-confluent U2OS (human osteosarcoma) and HBL100 (human mammary epithelial) cells were seeded onto 96-well plates, treated with neocarzinostatin (NCS, a radiomimetic reagent), doxorubicin, or hydroxyurea $(\mathrm{HU})$ for $20 \mathrm{~min}$ to $5 \mathrm{~h}$, and ROS levels were quantified using the fluorogenic dye DCFH$\mathrm{DA}$ and a fluorescence microplate reader. ${ }^{31}$ Although there were some differences in the amplitudes and kinetics of ROS induced by NCS, doxorubicin, and HU, all three compounds induce ROS biphasically in this time course, with an early ROS burst occurring within $2 \mathrm{~h}$ of DNA damage, a transient drop within $2-3.5 \mathrm{~h}$, followed by a second ROS burst occurring within $3.5-5 \mathrm{~h}$ of DNA damage (Figure $1 \mathrm{a}$ ).

ROS induces cell death after DNA damage. We tested if DNA-damage-induced ROS is important for the induction of

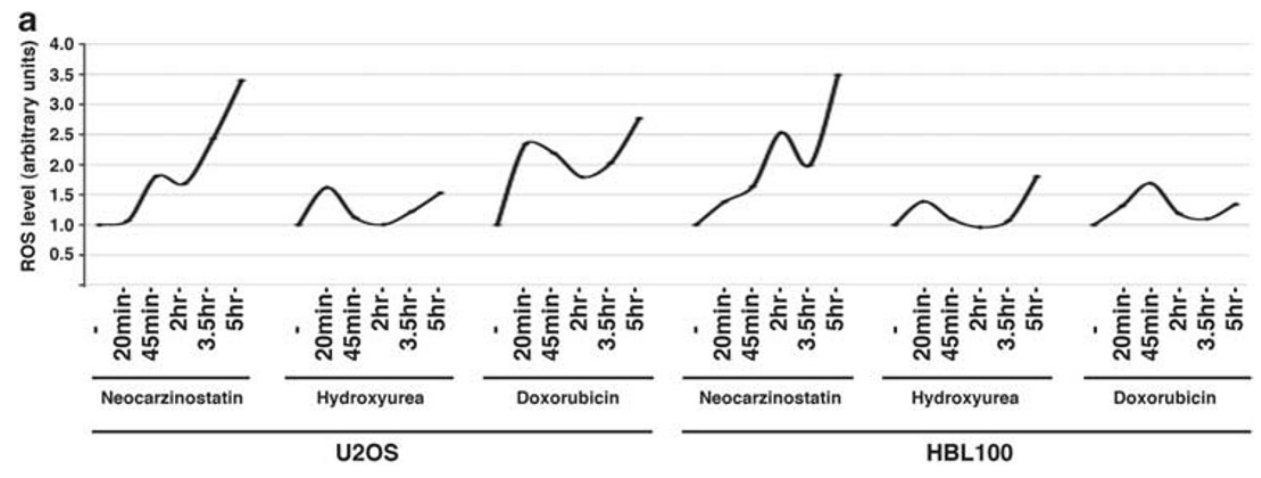

b

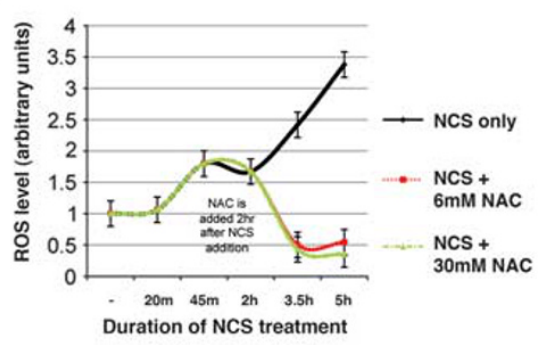

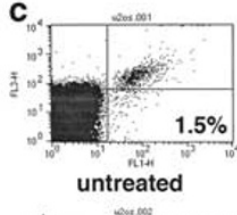

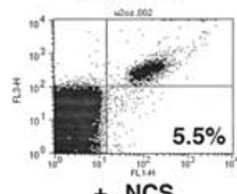

$+\mathrm{NCS}$

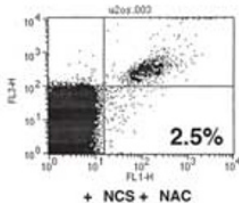

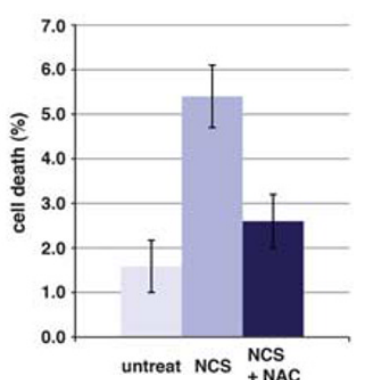

untreat NCS $\begin{aligned} & \text { NCS } \\ & + \text { NAC }\end{aligned}$

Figure 1 Exposure to DNA-damaging agents leads to increased levels of ROS that contribute to cell death. (a) Sub-confluent U2OS and HBL100 cells were treated with the DNA-damaging agents neocarzinostatin (NCS, $0.5 \mu \mathrm{g} / \mathrm{ml}$ ), hydroxyurea $(\mathrm{HU}, 2 \mathrm{mM})$, and doxorubicin $(8.5 \mu \mathrm{M})$ for $20 \mathrm{~min}$ to $5 \mathrm{~h}$. ROS levels were quantified using DCFHDA and a fluorescent microplate reader. (b) Addition of 6 or $30 \mathrm{mM} \mathrm{NAC} 2 \mathrm{~h}$ after NCS treatment suppressed the latent NCS-induced increase in ROS. (c) Suppression of the latent NCS-induced ROS by NAC treatment reduced NCS-induced apoptosis 
apoptosis. To study this, we suppressed the second NCSinduced ROS burst by adding the antioxidant NAC $2 \mathrm{~h}$ after NCS treatment, and quantified total cell death $24 \mathrm{~h}$ later. NCS induces DNA damage through the generation of free radicals, $^{32,33}$ resulting in the activation of DDR components, such as ATM, H2AX, p53, and DNA-PK. As expected, adding NAC immediately after NCS addition prevented both ROS production and the DNA-damageinduced activation of DDR proteins (data not shown). Meanwhile, adding NAC $2 \mathrm{~h}$ after NCS treatment abrogated the second burst of NCS-induced ROS without interfering with DDR activation (Figure 1b), allowing us to more accurately determine the effect of NCS-induced ROS on apoptosis induction. We found that suppression of the second NCS-induced ROS burst by NAC treatment significantly reduces cell death (Figure 1c). These results indicate that, once DDR proteins are activated immediately after DNA damage, an oscillation of increased ROS is generated, and this is crucial for subsequent cell death. Of note, the second burst of ROS induction is much higher than the first when cells are treated with NCS (Figure 1a), although phosphorylation of H2AX and ATM at Ser1981 are significantly lower than those observed within $20 \mathrm{~min}$ after treatment (Figure 2).

DNA damage increases levels of protein and phosphorylation of histone H2AX. It has been well illustrated that histone $\mathrm{H} 2 \mathrm{AX}$ is involved in DNA stress pathways and is phosphorylated by ATM in response to cell stress, and that the phosphorylated form of $\mathrm{H} 2 \mathrm{AX}(\gamma \mathrm{H} 2 \mathrm{AX})$ colocalizes to sites of DNA lesions. ${ }^{4,11,34}$ We found that, when the U2OS human osteosarcoma cell line and the HBL100 human mammary epithelial cell line are treated with NCS, levels of histone H2AX protein are markedly increased within 20-45 min after treatment (nearly 2-3-fold in U2OS

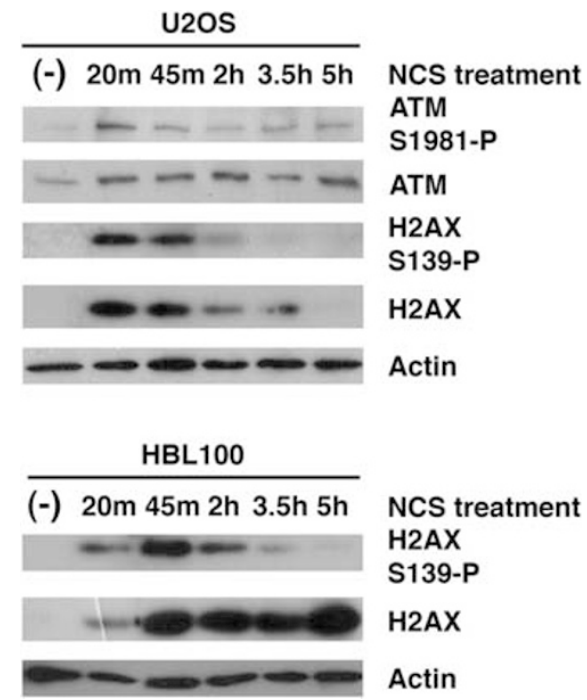

Figure 2 Upregulation and phosphorylation of $\mathrm{H} 2 \mathrm{AX}$ is an early event in the DDR cascade. U2OS and HBL100 cells were treated with NCS $(0.5 \mu \mathrm{g} / \mathrm{ml})$, for $20 \mathrm{~min}$ to $5 \mathrm{~h}$ in PBS buffer (to mimic the condition of cells used for ROS assays). Levels and phosphorylation of $\mathrm{H} 2 \mathrm{AX}$ and ATM protein were assayed by immunoblot analysis using antibodies indicated and HBL100 cells, respectively; Figure 2 and Supplementary Figure 1). An increase in $\mathrm{H} 2 \mathrm{AX}$ protein levels was also observed when cells were treated with doxorubicin (data not shown). When $\gamma \mathrm{H} 2 \mathrm{AX}$ levels were normalized to $\mathrm{H} 2 \mathrm{AX}$ protein levels through densitometric analysis, we found that NCS treatment did not increase $\mathrm{H} 2 \mathrm{AX}$ phosphorylation in U2OS or HBL100 cells (Supplementary Figure 1). Of note, upregulation of $\mathrm{H} 2 \mathrm{AX}$ after NCS treatment preceded that of ROS (Figures 1 and 2), raising the possibility that H2AX may mediate ROS induction after DNA damage.

Histone H2AX mediates ROS induction after DNA damage. By using U2OS and HBL100 cell lines, we established stable cell lines either overexpressing Flagtagged $\mathrm{H} 2 \mathrm{AX}$ (Flag-H2AX), or expressing a tet-regulatable H2AX shRNA in which the level of endogenous H2AX is decreased in the presence of doxycycline in cell culture. Using these cell lines, we tested whether levels of H2AX determine the basal or NCS-induced levels of ROS. Levels of Flag-H2AX and decreased levels of endogenous H2AX by shRNA were confirmed by immunoblot analysis. We found that, when H2AX was highly expressed in U2OS and HBL100 cells, basal levels of ROS were significantly increased, and that ROS was further produced when cells were treated with NCS, when compared with the levels of ROS in control U2OS and HBL100 cells (Figures $3 a$ and b).

Next, we knocked down endogenous histone H2AX by treating cells expressing tet-regulatable H2AX shRNA with doxycycline. Although basal levels of ROS did not change when endogenous H2AX was knocked down, induction of ROS by NCS was significantly attenuated (Figures $3 c$ and d). Together, the results from our H2AX overexpression and knockdown experiments provide strong evidence that $\mathrm{H} 2 \mathrm{AX}$ can regulate $\mathrm{ROS}$ levels.

Histone H2AX upregulates DNA-damage-induced ROS through Nox1 and Rac1. We investigated the mechanism by which histone H2AX increased the levels of ROS after DNA damage. Several proteins and pathways have been implicated in ROS production in mammalian cells. Among them are the $\operatorname{NADP}(\mathrm{H})$ oxidase (Nox) family of proteins: membrane-associated, multimeric flavin-containing enzymes that catalyze the reduction of oxygen using electrons from NADPH. A number of regulatory subunits have been identified for the Nox proteins, including p22 ${ }^{\text {phox }}$, NoxO1, NoxA1, and Rac1. ${ }^{13-15,17,35,36}$ When parental U2OS cells were treated with NCS for $5 \mathrm{~h}$, levels of ROS increased about 1.5-fold, whereas U2OS cells expressing Flag-H2AX exhibited higher induction of ROS compared with untreated U2OS cells expressing Flag-H2AX ( 1.8-fold, Figure 4a). However, when parental and Flag-H2AX expressing U2OS cells were treated with NCS in the presence of $0.5 \mu \mathrm{M}$ diphenyliodonium chloride (DPI, a broad-spectrum inhibitor of flavin-containing enzymes including Noxes), the increase in ROS was almost completely inhibited.

Inhibition of NCS-induced increases in ROS by DPI was also observed in HBL100 cells (Figure 4b). Levels of ROS in control HBL100 cells were increased $\sim 3.5$-fold with NCS treatment. When Flag-H2AX was expressed in HBL100 cells, induction of ROS was further increased $\sim 4$.3-fold compared 

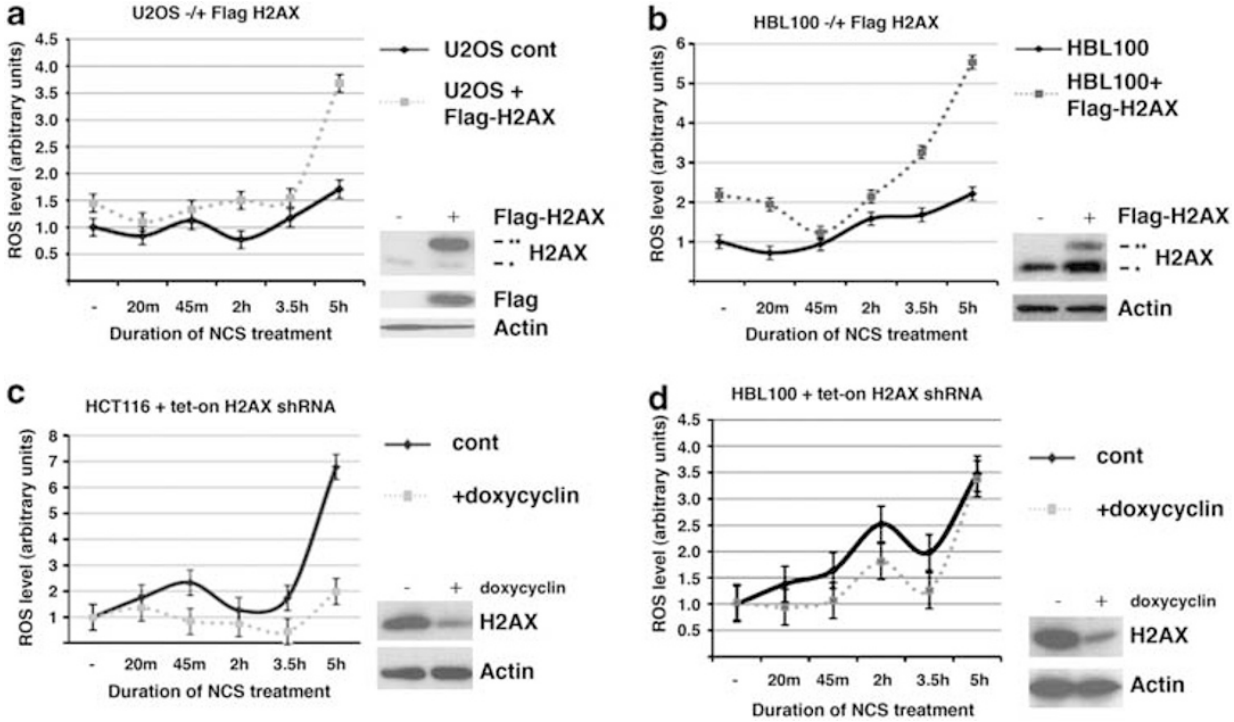

Figure 3 Histone H2AX increases basal and DNA damage-induced ROS. Overexpression of Flag-H2AX increased basal and NCS-induced ROS in U2OS (a) and HBL-100 (b) cells, whereas inducible H2AX knockdown reduces NCS-induced ROS in HCT-116 (c) and HBL100 (d) cells. Immunoblot analysis was performed at $5 \mathrm{~h}$ after NCS treatment. **Flag-tagged H2AX, ${ }^{*}$ endogenous H2AX
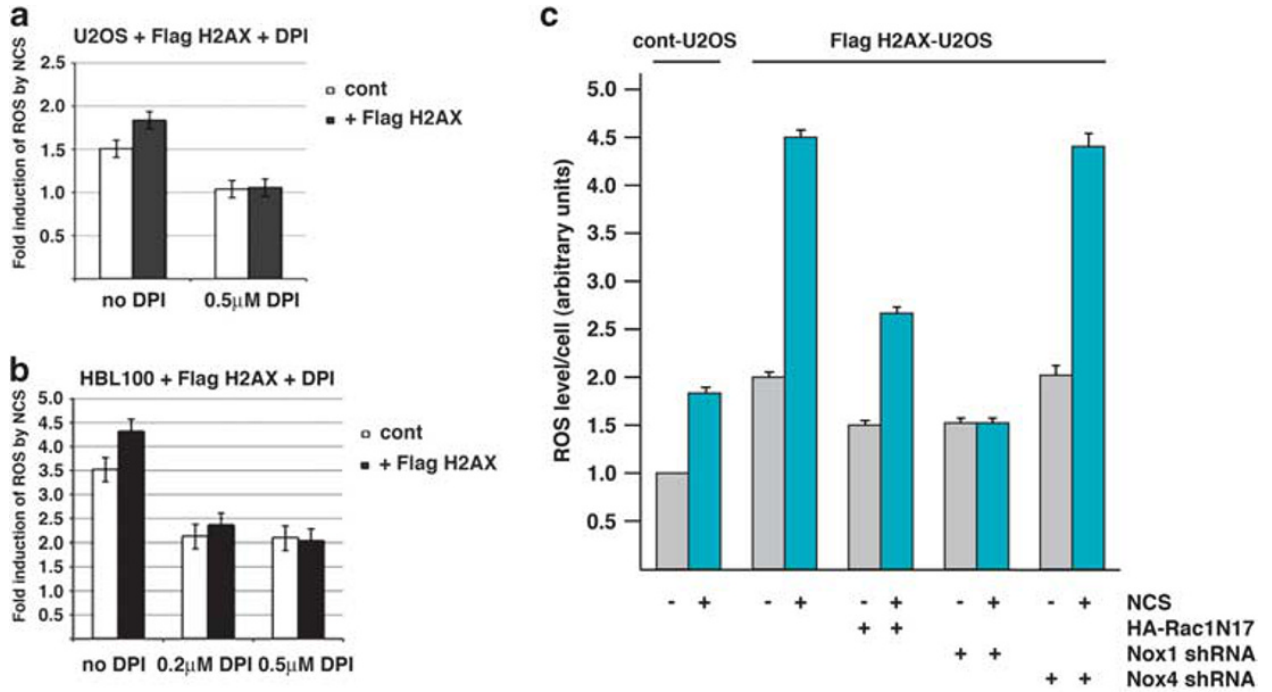

e
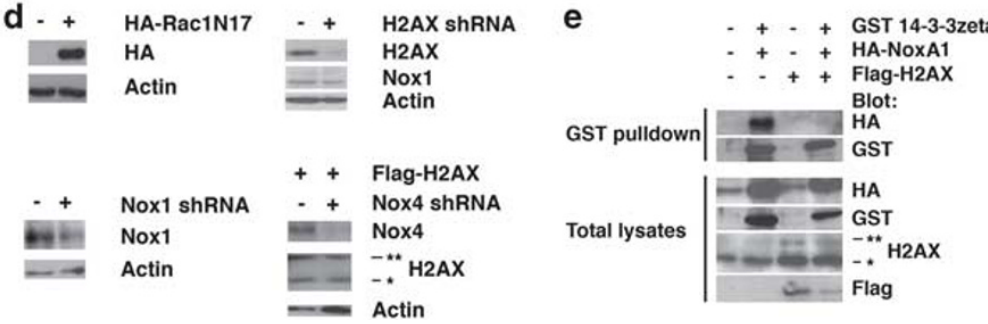

Figure 4 H2AX increases ROS through Nox1/Rac1 pathway. Control U2OS cells (a, white bar), Flag-H2AX U2OS cells (a, black bar), control HBL100 cells (b, white bar), and Flag-H2AX HBL100 cells (b, black bar) were treated with NCS $(0.5 \mu \mathrm{g} / \mathrm{ml}, 5 \mathrm{~h})$ with or without the Nox-inhibitor, DPI $(0.5 \mu \mathrm{M})$ and fold-induction of ROS was measured. (c) Vector control cells or Flag-H2AX U2OS cells were transiently transfected with an indicated plasmid with a control vector for $12 \mathrm{~h}$, followed by NCS treatment $(0.5 \mu \mathrm{g} / \mathrm{ml})$ for $5 \mathrm{~h}$. Levels of ROS were measured in more than three independent experiments. (d) Levels of proteins were immunoblotted to confirm expression or knockdown of indicated proteins. (e) Expression of H2AX inhibited coimmunoprecipitation of 14-3-3zeta with NOXA1. U2OS cells were transfected with the indicated plasmids, followed by GST-pulldown of 14-3-3zeta. **Flag-taged H2AX, "endogenous H2AX 
with untreated HBL100 cells expressing Flag-H2AX (Figure 4b). Two different concentrations of DPI (0.2 and $0.5 \mu \mathrm{M}$ ) were examined; both concentrations inhibited ROS production in control HBL100 cells and HBL100 cells expressing Flag-H2AX. Of note, DPI did not completely inhibit ROS production in these cell lines at these concentrations, which suggests that Nox-independent mechanisms also produce ROS in HBL100 cells.

These results strongly suggest that $\mathrm{H} 2 \mathrm{AX}$ induces ROS by activating Nox protein(s) when cells are exposed to DNAdamaging agents. We then sought to identify the Nox enzyme that mediates H2AX-induced ROS. Nox1 and Nox4 are the two most ubiquitous Nox enzymes in epithelial and endothelial cells, ${ }^{34}$ whereas Nox2 is highly expressed in phagocytes. ${ }^{37}$ It has been demonstrated that Nox1 activity is Rac1-dependent, ${ }^{13,18}$ whereas Nox4 activity is not. ${ }^{37,38}$ Based on these observations, we used the pSilencer4.1-CMV-neo vector to generate a plasmid expressing Nox1 shRNA and examined the effect of Nox1 knockdown on DNA-damage-induced ROS in H2AX overexpressing cells. In vector control U2OS cells, NCS treatment increased ROS 1.7-fold. Basal levels of ROS in U2OS cells expressing Flag-H2AX were higher ( $\sim 2.0$-fold) than U2OS vector control cells; this was further increased $\sim 4$.4-fold when cells were treated with NCS. We found that Nox1 knockdown with shRNA similarly reduced both basal and DNA-damage-induced ROS in U2OS cells expressing Flag-H2AX to $\sim 1.5$-fold (Figure $4 \mathrm{c}$ ). Of note, this is still higher than control, suggesting that DNA damage also induces ROS through pathways that do not involve the H2AXNox1 axis (see Figure 5). Consistent with these observations, when Nox1 is knocked down in NCS-treated parental U2OS and 293T cells, ROS were still produced (Supplementary Figure 4). On the other hand, when Nox4 is knocked down in U2OS cells expressing Flag-H2AX, no significant decrease in the levels of basal and NCS-induced ROS was observed (Figure 4c).

To further investigate whether the Nox1 pathway was involved in $\mathrm{H} 2 \mathrm{AX}$-mediated ROS induction, we introduced a dominant negative form of Rac1 (Rac1N17) in parental and Flag-H2AX-overexpressing U2OS cells to inhibit the Rac1 GTPase, which is necessary for the activation of Nox $1 .^{13}$ When Rac1N17 was co-expressed in Flag-H2AX U2OS cells, basal and NCS-induced levels of ROS were decreased to $\sim 1.5$-fold and $\sim 2.6$-fold, respectively, compared with the untreated Flag-H2AX U2OS cells (Figure 4c). Knockdown of Nox1 also reduced basal levels of ROS in those cells (Supplementary Figure 2). Levels of the proteins studied in these experiments were shown in Figure $4 \mathrm{~d}$.

We then explored the mechanisms of how increased H2AX increases the levels of ROS. Recent studies have demonstrated that 14-3-3zeta binds to NoxA1, resulting in the inactivation of Nox 1 by sequestration of NoxA1 from Nox 1 in the plasma membrane. ${ }^{27}$ Interestingly, more recent proteomic analysis indicated that $\mathrm{H} 2 \mathrm{AX}$ constitutively binds to 14-33zeta in a normally growing human hepatocellular carcinoma cell line. ${ }^{28}$ It is therefore possible that DNA damage-induced H2AX titrates out the 14-3-3zeta from NoxA1, thus potentially increasing the formation of an active Nox1/NoxA1 complex. We found that GST-tagged 14-3-3zeta co-immunoprecipitates a complex containing HA-tagged NoxA1 in control U2OS

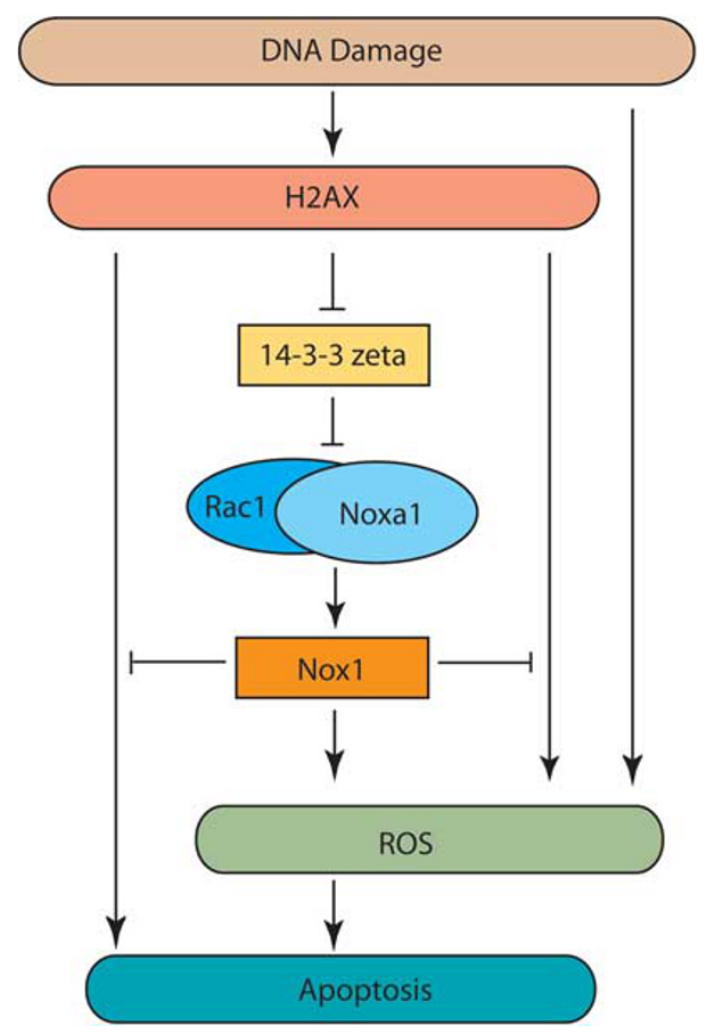

Figure 5 Model of ROS induction by H2AX after DNA damage. DNA damage induces levels and phosphorylation of H2AX. Then, H2AX activates Rac1 GTPase and Nox1 by sequestering 14-3-3zeta, leading to increase in intracellular levels of ROS. H2AX can also induce ROS and apoptosis by the alternative pathways that are usually inhibited by Nox1

cells. However, this complex was not detected in Flag-H2AX U2OS cells (Figure 4e). Thus, we postulate that overexpression of H2AX causes NoxA1 to be released from 14-3-3zeta resulting in an increase in ROS levels.

It has been illustrated that EGF stimulation or oncogenic signals such as K-RasVal12 upregulates Nox1 mRNA. ${ }^{19}$ In light of this, we tested whether the activation of Nox1 signaling by $\mathrm{H} 2 \mathrm{AX}$ occurs through an upregulation in Nox1 protein levels. However, we found that neither $\mathrm{H} 2 \mathrm{AX}$ overexpression nor H2AX depletion by shRNA causes a significant change in Nox1 protein levels (not shown), suggesting that the activation of Nox1 pathway by $\mathrm{H} 2 \mathrm{AX}$ is not through an increase in the levels of Nox1 protein.

These results indicate that the increased level of $\mathrm{H} 2 \mathrm{AX}$ in DNA-damaged cells promotes the activation of the Nox1/ Rac1 pathway and ultimately increases intracellular levels of ROS.

H2AX-mediated apoptosis is inhibited by Rac1N17. It has been shown that high levels of ROS can induce apoptosis. ${ }^{22}$ As our results demonstrate that expression of $\mathrm{H} 2 \mathrm{AX}$ increases the levels of ROS, we tested whether H2AX can induce apoptosis without DNA stress. Constitutive levels of apoptosis were determined in control and Flag-H2AX U2OS cells. As shown in Figure 6a, levels of apoptosis are 

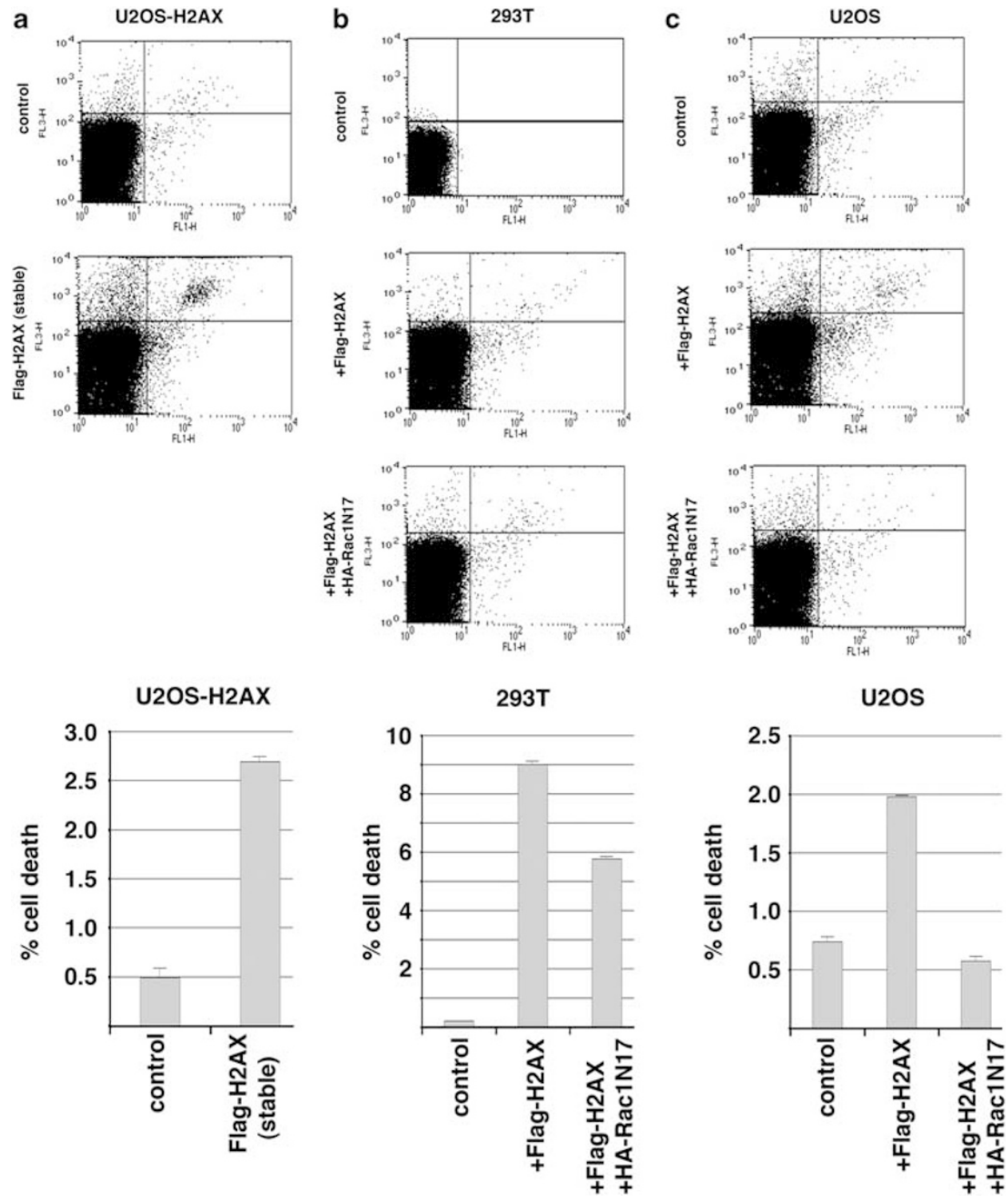

Figure 6 High levels of H2AX induces apoptosis without DNA damage, which is decreased by Rac1N17. (a) U2OS cells stably expressing Flag-H2AX show constitutive apoptosis without DNA damage, compared with vecetor control cells. 293T (b) or U2OS (c) cells were transfected with expression vectors of Falg-H2AX, HA-Rac1N17 or both for $48 \mathrm{~h}$, and apoptosis was compared with that of vector control cells. A bar graph represents fraction of apoptotic cells

significantly elevated in U2OS cells stably expressing FlagH2AX. Increased levels of apoptosis were also studied in 293T and U2OS cells transiently co-expressing Flag-H2AX and HA-Rac1N17 (Figures $6 \mathrm{~b}$ and c). In both cell types, expression of Flag-H2AX significantly increased the level of apoptosis, whereas co-expression of HA-Rac1N17 reduced Flag-H2AX-induced apoptosis. Inhibition of apoptosis by HA-Rac1N17 was less effective in 293T cells than in U2OS cells, suggesting that regulation of apoptosis by H2AX/Rac1 may be determined by cell context.

\section{Discussion}

DNA damage from various sources has been shown to increase intracellular levels of ROS, ${ }^{20}$ which in turn regulates cell death or senescence through p53-dependent and -independent mechanisms. ${ }^{22}$ Induction of cell death or senescence is one of the most important defense responses against malignant transformation. A well-characterized pathway of ROS production and regulation is through the p53 tumor suppressor and the mitochondria. ${ }^{21,24}$ However, the detailed mechanism of ROS generation after DNA damage remains to be uncovered and the roles of other DDR proteins in ROS regulation still need to be investigated.

Our studies indicate that DNA damage-induced apoptosis is reduced when the second burst of DNA damage-induced ROS is suppressed by treatment of cells with the antioxidant NAC. Autophosphorylation of ATM at Ser1981 occurred within 20 min of NCS treatment. On the other hand, an increase in $\mathrm{H} 2 \mathrm{AX}$ protein and its phosphorylation was initially observed within 20 min after NCS treatment and reached its peak within $45 \mathrm{~min}$ in U2OS and HBL100 cell lines. We found 
that the second burst of ROS induction (3.5-5h after NCS in U2OS and HBL100 cells, Figure 1a) was not accompanied by increased phosphorylation of ATM and H2AX (Figure 2, data not shown), indicating that the first burst, but not the second, of ROS induction within the first 20-45 min after DNA damage require the activation/phosphorylation of DDR proteins and the resultant apoptotic phenotype.

Although the importance of histone H2AX in the accumulation of DDR and repair proteins at sites of DSBs has been well established, ${ }^{4,5,9,12}$ the role of $\mathrm{H} 2 \mathrm{AX}$ in other signaling pathways is not fully understood. In this study, we established a novel pathway that DNA damage increases intracellular ROS levels through histone $\mathrm{H} 2 \mathrm{AX}, \mathrm{Rac1}$, and Nox1. We found that levels of intracellular ROS are increased by not only DNA damage but also $\mathrm{H} 2 \mathrm{AX}$ overexpression. Given that DNA damage increases endogenous levels of ROS, ${ }^{24-26}$ our results suggest a signaling loop between ROS production and increase in $\mathrm{H} 2 \mathrm{AX}$ to amplify the signal causing apoptosis.

In the cell lines we tested, DNA damage induces ROS biphasically, with an early burst occurring within $45 \mathrm{~min}$ of DNA damage induction and a late second burst occurring within $5 \mathrm{~h}$. The late burst of ROS induced by NCS can be inhibited by either Nox1 shRNA or Rac1N17 (see Supplementary Figure 3). However, the early burst of ROS is not inhibited by Nox1 shRNA or Rac1N17, suggesting that whereas the late burst of ROS is generated through the Nox1/Rac1 axis, the early burst is induced through DDR components. Furthermore, when Rac1/Nox1 is inhibited, cells still produce certain levels of ROS (Figure 4c), suggesting that production of ROS is at least in part amplified in Rac1/Nox1independent manner.

We found that overexpression or knockdown of H2AX does not significantly change Nox1 protein levels (Figure 4, not shown), suggesting that H2AX does not increase ROS levels by augmenting Nox1 protein expression or stability. However, coimmunoprecipitation assays indicate that the Nox1 activator NoxA1 is released from 14-3-3zeta in cells expressing high levels of $\mathrm{H} 2 \mathrm{AX}$. As it has been shown that binding of 14-3-3zeta to NoxA1 complex is inhibitory for Nox1 activity, ${ }^{27}$ we propose a model where increased $\mathrm{H} 2 \mathrm{AX}$ binds and sequester cellular 14-3-3zeta, resulting in the release of NoxA1 and the activation of Nox1 complex.

There are some potential links between our findings and human diseases. Constitutive production of superoxide was induced upon Ras transformation in NIH3T3 cells, and depletion of its dismutated metabolite $\mathrm{H}_{2} \mathrm{O}_{2}$ suppressed the uncontrolled growth of Ras-transformed cells. ${ }^{39}$ Significantly, introduction of Nox1 siRNA into K-RasVal12-transformed NRK (normal rat kidney) cells was shown to block their anchorageindependent growth and induced morphological reversion, ${ }^{19}$ supporting the idea that Nox1-mediated production of ROS has crucial roles in cell transformation. Our observation that $\mathrm{H} 2 \mathrm{AX}$ can activate Nox1/Rac1-mediated production of ROS raises the possibility that $\mathrm{H} 2 \mathrm{AX}$ may be involved in cell transformation induced by Ras and other oncogenes.

It has been shown recently that the status of $\mathrm{H} 2 \mathrm{AX}$ phosphorylation is crucial to determine whether cells will survive or undergo apoptosis after DNA damage. ${ }^{7}$ Because ROS can also activate both cell survival and apoptotic pathways depending on its concentration and localization, ${ }^{14,21,37}$ regulation of
ROS by H2AX may be one mechanism to determine cell survival or apoptosis after DNA insults. Therefore, elucidating the relationship between $\mathrm{H} 2 \mathrm{AX}$ phosphorylation status, ROS regulation, and cell fate after DNA damage may provide understanding of DDR regulation relevant to the inhibition of cancer growth and progression.

\section{Materials and Methods}

Cell culture. U2OS, HBL100, HeLa, and HCT-116 cells were maintained in Dulbecco's Modified Eagle Medium (GIBCO, Grand Island, NY, USA), supplemented with 10\% fetal bovine serum (Invitrogen, Grand Island, NY, USA) or cosmic calf serum (HyClone, Logan, UT, USA). To induce DNA damage, cells were treated with $0.5-1 \mu \mathrm{g} / \mathrm{ml}$ neocarzinostatin (Kayaku, Tokyo, Japan), $8.5 \mu \mathrm{M}$ doxorubicin (Sigma, St. Louis, MO, USA), or $2 \mathrm{mM}$ hydroxyurea (Sigma).

Plasmids and transfection. pSingle-tTs-shRNA vector (Clontech, Mountain View, CA, USA) was used to generate plasmids expressing tet-inducible $\mathrm{H} 2 \mathrm{AX}$ shRNA with the target sequence $5^{\prime}$-CTGGAATTCTGCAGCTAAC- $3^{\prime}$ or $5^{\prime}$-CAACAAGAAGACGCGAATC-3', according to the manufacturer's instructions. Briefly, shRNA-encoding oligonucleotides were synthesized, annealed, and cloned into Xho1/Hindlll sites of the vector. pcDNA3-Flag-H2AX was generated by subcloning Flag-H2AX from pEF into the EcoR1/Not1 sites of pcDNA3. pEF-HARac1WT/Rac1N17 was generated by PCR amplification from a complementary DNA library, followed by site-directed mutagenesis (Stratagene, Wilmington, DE, USA) and subsequent subcloning into a pEF vector. pEBG-14-3-3zeta was purchased from Addgene (Cambridge, MA, USA). Nox1 complementarty DNA in pcDNA3 was obtained from Drs. Banfi Botond (University of lowa) and Hideki Sumimoto (Kyushu University, Japan). pSilencer4.1-CMV-Neo vector was used to generate plasmids expressing Nox1 and Nox4 shRNA with the target sequences $5^{\prime}$-CGACAGTGGAGTATGTGAC-3'(Nox1) and 5'-TTATTGCATATGTAGAGGCTC TGAT-3' (Nox4), according to the manufacturer's instructions. Briefly, shRNAencoding oligonucleotides were synthesized, annealed, and cloned into HindIII/ $\mathrm{BamH} 1$ sites of the vector. Transfections were performed with Fugene- 6 or FugeneHD (Roche Applied Science, Indianapolis, IN, USA), according the manufacturer's protocol.

Generation of stable cell lines. To generate stable H2AX-overexpression and tet-on-H2AX-knockdown cell lines, cells were first transfected with pcDNA3Flag-H2AX and pSingle-tTS-H2AX-shRNA. Forty-eight to seventy-two hours after transfection, cells were reseeded at low density and stable transfectants were positively selected using neomycin at pre-optimized concentrations $(1000 \mu \mathrm{g} / \mathrm{ml}$ for U2OS, $500 \mu \mathrm{g} / \mathrm{ml}$ for HCT116, and $1500 \mu \mathrm{g} / \mathrm{ml}$ for HBL100). Stable transfectants were cultured in neomycin-containing media until colonies become visible (after approximately 1-2 weeks in culture); media (and neomycin) is changed and refreshed every $72 \mathrm{~h}$. Colonies were trypsinized and transferred to 96-well or 48-well plates when they become visible, and both colonies and 'pools' of stable transfectants (a pool of the colonies that were not picked) were cultured for an additional 1-2 weeks. Stable transfectants were subsequently screened for H2AX overexpression and knockdown through western blot. Inducible H2AX knockdown was performed by culturing cells stably expressing pSingle-tTS-H2AX-shRNA in media containing $2 \mu \mathrm{g} / \mathrm{ml}$ doxycycline (Sigma) for $24 \mathrm{~h}$ (HCT116) or $48 \mathrm{~h}$ (HBL100).

Immunoblot analysis and densitometry analysis. The following primary antibodies were purchased for immunoblot analysis: for H2AX protein (77635, Genetex, Irvine, CA, USA), $\alpha$-tubulin (5286, Santa Cruz Biotechnology, Santa Cruz, CA, USA), $\beta$-actin (C-11, Santa Cruz Biotechnology), HA-probe (Y-11, Santa Cruz Biotechnology). Nox1 antibody (Abcam, Cambridge, MA, USA) was a generous gift from Dr. Andrean Simons. Cell extracts were prepared in EBC buffer ( $50 \mathrm{mM}$ HEPES, pH 7.6, $250 \mathrm{mM} \mathrm{NaCl}, 0.1 \%$ Nonidet P-40, $5 \mathrm{mM}$ EDTA, pH 8.0, with mixed protease inhibitor (aprotinin, leupeptin, PMSF; Sigma). The secondary antibodies (Jackson Immuno-laboratory, Mill Valley, CA, USA) were peroxidaseconjugated anti-mouse $\lg G(\mathrm{H}+\mathrm{L})$, anti-rabbit $\lg G(\mathrm{H}+\mathrm{L})$, or anti-goat $\lg G$ $(\mathrm{H}+\mathrm{L})$. Film (Hyblot, Denville Scientific, Metuchen, $\mathrm{NJ}, \mathrm{USA}$ ) was developed using ECL (Enhanced ChemiLuminescence) reagent. The level of phosphorylation and expression of proteins were measured by densitometry using ImageJ software (National Institutes of Health) and the data were expressed as arbitrary units relative to band intensity of total protein or internal control. 
Quantifying ROS levels. ROS levels were measured using 2,7dichlorodihydrofluorescein diacetate oxidation (DCFH-DA, Sigma) and a fluorescence microplate reader. ${ }^{31}$ Briefly, cells were seeded onto 96-well plates (black/clear bottom, BD Biosciences, San Jose, CA, USA) at a density of 500010000 cells per well. Twenty-four hours after seeding (at $40-70 \%$ density), cells were washed with PBS buffer (Invitrogen), treated with $100 \mu \mathrm{M} \mathrm{DCFH-DA} \mathrm{(diluted} \mathrm{in}$ DMEM $+1 \% \mathrm{FBS}$ ) for $1 \mathrm{~h}\left(\right.$ at $\left.37^{\circ} \mathrm{C}, 5 \% \mathrm{CO}_{2}\right)$. Cells were then washed with PBS again, and treated with the DNA-damaging agents NCS, doxorubicin, or hydoryurea (diluted in PBS) for $20 \mathrm{~min}-5 \mathrm{~h}$. DCF fluorescence is measured using a fluorescent microplate reader (Spectramax, Sunnyvale, CA, USA). Readings were done in triplicates, quadruplicates, or quintuplicate. ROS levels were normalized to cell number, which was measured using a fluorogenic DNA-binding dye (CyQuant Proliferation assay kit, Molecular Probes (Eugene, OR, USA), Invitrogen), and ROS per cell levels were quantified by dividing DCF fluorescence with CyQuant fluorescence. Because DCFH-DA and CyQuant have the same excitation/emission spectra, we stained cells with DCFH-DA and CyQuant in different (exact replicate) wells. Error bars represent the average standard error of all the samples in a single experiment.

Cell death assays. Apoptosis was quantified using the Annexin V-FITC Apoptosis Detection Kit (Calbiochem, San Diego, CA, USA) and a FACScalibur flow cytometer (BD Biosciences), according to the manufacturer's instructions. Twentyfour hours after seeding, NCS was added to cell culture media. NAC (Sigma) was added $2 \mathrm{~h}$ after NCS addition, and Annexin-V-FITC assays were performed $24 \mathrm{~h}$ later. Apoptosis of $293 \mathrm{~T}$ and U2OS cells after transient transfection of Flag-H2AX/pcDNA3 or HA-Rac1N17/pEF was determined at $48 \mathrm{~h}$.

\section{Conflict of Interest}

The authors declare no conflict of interest.

Acknowledgements. We thank Drs. Banfi Botond (University of lowa) and Hideki Sumimoto (Kyushu University, Japan) for sharing Nox1 cDNA. This work was supported in part by NIH Grants K01CA134941 (ALS), R01CA133114 (DRS), and R01CA79892, R01CA90631, Susan G Komen Breast Cancer Research Fund and Avon Breast Cancer Fund (TO).

1. Bassing $\mathrm{CH}$, Alt $\mathrm{FW}$. The cellular response to general and programmed DNA double strand breaks. DNA Repair 2004; 3: 781-796.

2. Zhou BB, Elledge SJ. The DNA damage response: putting checkpoints in perspective. Nature 2000; 408: 433-439.

3. Ciccia A, Elledge SJ. The DNA damage response: making it safe to play with knives. $\mathrm{Mol}$ Cell 2010; 40: 179-204.

4. Fernandez-Capetillo $O$, Lee A, Nussenzweig M, Nussenzweig A. H2AX: the histone guardian of the genome. DNA Repair 2004; 3: 959-967.

5. Stucki M, Clapperton JA, Mohammad D, Yaffe MB, Smerdon SJ, Jackson SP. MDC1 directly binds phosphorylated histone $\mathrm{H} 2 \mathrm{AX}$ to regulate cellular responses to DNA doublestrand breaks. Cell 2005; 123: 1213-1226.

6. Bassing $\mathrm{CH}$, Suh H, Ferguson DO, Chua KF, Manis J, Eckersdorff M et al. Histone H2AX: a dosage-dependent suppressor of oncogenic translocations and tumors. Cell 2003; 114: 359-370.

7. Cook PJ, Ju BG, Telese F, Wang X, Glass CK, Rosenfeld MG. Tyrosine dephosphorylation of $\mathrm{H} 2 \mathrm{AX}$ modulates apoptosis and survival decisions. Nature 2009; 458: 591-596.

8. Stiff T, O'Driscoll M, Rief N, Iwabuchi K, Lobrich M, Jeggo PA. ATM and DNA-PK function redundantly to phosphorylate $\mathrm{H} 2 \mathrm{AX}$ after exposure to ionizing radiation. Cancer Res 2004; 64: 2390-2396.

9. Paull TT, Rogakou EP, Yamazaki V, Kirchgessner CU, Gellert M, Bonner WM. A critical role for histone $\mathrm{H} 2 \mathrm{AX}$ in recruitment of repair factors to nuclear foci after DNA damage. Curr Biol 2000; 10: 886-895.

10. Rogakou EP, Boon C, Redon C, Bonner WM. Megabase chromatin domains involved in DNA double-strand breaks in vivo. J Cell Biol 1999; 146: 905-916.

11. Rogakou EP, Pilch DR, Orr AH, Ivanova VS, Bonner WM. DNA double-stranded breaks induce histone H2AX phosphorylation on serine 139. J Biol Chem 1998; 273: 5858-5868.

12. Huen MS, Grant R, Manke I, Minn K, Yu X, Yaffe MB et al. RNF8 transduces the DNAdamage signal via histone ubiquitylation and checkpoint protein assembly. Cell 2007; 131: 901-914.
13. Cheng G, Diebold BA, Hughes $Y$, Lambeth JD. Nox1-dependent reactive oxygen generation is regulated by Rac1. J Biol Chem 2006; 281: 17718-17726.

14. Lambeth JD. NOX enzymes and the biology of reactive oxygen. Nat Rev Immunol 2004; 4: 181-189.

15. Lambeth JD, Kawahara T, Diebold B. Regulation of Nox and Duox enzymatic activity and expression. Free Radic Biol Med 2007; 43: 319-331.

16. Ambasta RK, Kumar P, Griendling KK, Schmidt HH, Busse R, Brandes RP. Direct interaction of the novel Nox proteins with p22phox is required for the formation of a functionally active NADPH oxidase. J Biol Chem 2004; 279: 45935-45941.

17. Banfi B, Clark RA, Steger K, Krause KH. Two novel proteins activate superoxide generation by the NADPH oxidase NOX1. J Biol Chem 2003; 278: 3510-3513.

18. Miyano K, Ueno N, Takeya R, Sumimoto H. Direct involvement of the small GTPase Rac in activation of the superoxide-producing NADPH oxidase Nox1. J Biol Chem 2006; 281: 21857-21868.

19. Mitsushita J, Lambeth JD, Kamata T. The superoxide-generating oxidase Nox1 is functionally required for Ras oncogene transformation. Cancer Res 2004; 64: 3580-3585.

20. Rowe LA, Degtyareva N, Doetsch PW. DNA damage-induced reactive oxygen species (ROS) stress response in Saccharomyces cerevisiae. Free Radic Biol Med 2008; 45: 1167-1177.

21. Hamanaka RB, Chandel NS. Mitochondrial reactive oxygen species regulate cellular signaling and dictate biological outcomes. Trends Biochem Sci 2010; 35: 505-513.

22. Simon HU, Haj-Yehia A, Levi-Schaffer F. Role of reactive oxygen species (ROS) in apoptosis induction. Apoptosis 2000; 5: 415-418.

23. Bragado $P$, Armesilla A, Silva A, Porras A. Apoptosis by cisplatin requires p53 mediated p38alpha MAPK activation through ROS generation. Apoptosis 2007; 12: 1733-1742.

24. Liu B, Chen Y, St Clair DK. ROS and p53: a versatile partnership. Free Radic Biol Med 2008; 44: 1529-1535.

25. Polyak K, Xia Y, Zweier JL, Kinzler KW, Vogelstein B. A model for p53-induced apoptosis. Nature 1997; 389: 300-305

26. Sablina AA, Budanov AV, Ilyinskaya GV, Agapova LS, Kravchenko JE, Chumakov PM The antioxidant function of the p53 tumor suppressor. Nat Med 2005; 11: 1306-1313.

27. Kim JS, Diebold BA, Babior BM, Knaus UG, Bokoch GM. Regulation of Nox1 activity via protein kinase A-mediated phosphorylation of NoxA1 and 14-3-3 binding. $\mathrm{J}$ Biol Chem 2007; 282: 34787-34800.

28. Yang X, Zou P, Yao J, Yun D, Bao H, Du R et al. Proteomic dissection of cell type-specific $\mathrm{H} 2 \mathrm{AX}$-interacting protein complex associated with hepatocellular carcinoma. J Proteome Res 2010; 9: 1402-1415

29. Evert BA, Salmon TB, Song B, Jingjing L, Siede W, Doetsch PW. Spontaneous DNA damage in Saccharomyces cerevisiae elicits phenotypic properties similar to cancer cells. J Biol Chem 2004; 279: 22585-22594.

30. Salmon TB, Evert BA, Song B, Doetsch PW. Biological consequences of oxidative stress-induced DNA damage in Saccharomyces cerevisiae. Nucleic Acids Res 2004; 32: $3712-3723$

31. Wang H, Joseph JA. Quantifying cellular oxidative stress by dichlorofluorescein assay using microplate reader. Free Radic Biol Med 1999; 27: 612-616.

32. Goldberg $\mathrm{IH}$. Free radical mechanisms in neocarzinostatin-induced DNA damage. Free Radic Biol Med 1987; 3: 41-54.

33. Goldberg $\mathrm{IH}$. Mechanism of neocarzinostatin action: role of DNA microstructure in determination of chemistry of bistranded oxidative damage. Acc Chem Res 1991; 24: 191-198.

34. Rokutan K, Kawahara T, Kuwano Y, Tominaga K, Nishida K, Teshima-Kondo S. Nox enzymes and oxidative stress in the immunopathology of the gastrointestinal tract. Semin Immunopathol 2008; 30: 315-327.

35. Sumimoto $H$. Structure, regulation and evolution of Nox-family NADPH oxidases that produce reactive oxygen species. FEBS J 2008; 275: 3249-3277.

36. Sumimoto $\mathrm{H}$, Miyano K, Takeya R. Molecular composition and regulation of the Nox family NAD(P)H oxidases. Biochem Biophys Res Commun 2005; 338: 677-686.

37. Brown DI, Griendling KK. Nox proteins in signal transduction. Free Radic Biol Med 2009; 47: $1239-1253$.

38. Ellmark SH, Dusting GJ, Fui MN, Guzzo-Pernell N, Drummond GR. The contribution of Nox4 to NADPH oxidase activity in mouse vascular smooth muscle. Cardiovasc Res 2005; 65: 495-504

39. Irani K, Xia Y, Zweier JL, Sollott SJ, Der CJ, Fearon ER et al. Mitogenic signaling mediated by oxidants in Ras-transformed fibroblasts. Science 1997; 275: 1649-1652.

Cell Death and Disease is an open-access journal published by Nature Publishing Group. This work is licensed under the Creative Commons Attribution-Noncommercial-No Derivative Works 3.0 Unported License. To view a copy of this license, visit http://creativecommons.org/licenses/by-nc-nd/3.0/ 